\title{
CONFORMATIONAL STUDIES OF SOME NORMAL, MEDIUM AND LARGE RING SYSTEMS
}

\author{
J. DALe \\ Department of Chemistry, University of Oslo, Norway
}

\begin{abstract}
The following lines of research are discussed:

1. Equilibria between 5-, 6- and 7-membered heterocyclic rings have been established by competition reactions of polymethylene chains for ether oxygen or amino nitrogen.

2. Surprisingly high n.m.r. coalescence temperatures are found for cis,ciscyclodeca-1,6-diene and derivatives, and for several medium ring ketones carrying gem-dimethyl substituents. It is suggested that a low-barrier pseudorotation process becomes blocked by the introduction of double bonds and geminal substituents.

3. Macrocyclic diketones and hydrocarbons $\left(\mathrm{C}_{14}-\mathrm{C}_{24}\right)$ carrying gem-dimethyl groups in relative positions that fit all four allowed corner positions of rectangular 'diamond lattice' conformations have been synthesized. Conformational homogeneity is observed for all hydrocarbons, but only for the 14- and 16-membered diketone.

4. Cyclic oligopeptides of sarcosine have been synthesized. The n.m.r. spectra of the tri-, tetra-, penta- and octa-peptide indicate configurational (cis-trans) and conformational homogeneity. In spite of the impossibility of hydrogen bonding, there are indications of strong transannular interactions.

5. Cyclic dimethylsiloxanes up to the 32 -membered ring (hexadecamer) have been isolated from equilibrium mixtures. Chemical and physical properties do not permit conclusions about preferred conformations, if any.
\end{abstract}

IN THIS paper I shall try to present five separate lines of research. These may seem very different chemically, but have this in common that they are attempts to shed light on the conformational behaviour of ring systems. Our investigations may so far have been less penetrating than some of the others presented at this meeting, but have, perhaps, more the character of prospecting in new areas. Some of the studies have been successful and may point to an extension of the scope of conformational analysis; others have given less conclusive results and demonstrate certain limitations, in accord with both themes of this Symposium.

(1). The first part is concerned with attempts to establish equilibria between 'saturated' rings of different sizes, as a means of determining directly the balance between enthalpy and entropy effects. Thus, while a six-membered 
ring, having a single, unstrained chair conformation, is favoured by a low enthalpy, its entropy is for this same reason unfavourably small. On the other hand, the inherently strained five- and seven-membered rings have higher enthalpies, but gain much entropy by pseudorotation through a large number of similarly strained conformations. Such equilibria are of course well known in carbohydrate chemistry, but the stability of furanose and pyranose rings may be quite differently influenced by the numerous substituents.

To be able to establish equilibria between different ring sizes, one is limited to heterocyclic compounds. Since the ammonium group is known from many examples $^{1}$ to be conformationally equivalent to saturated carbon, it was attempted to equilibrate quaternary ammonium salts through a thermal mechanism involving ring opening by departure of an alkyl bromide group, subsequent re-alkylation of the free amine by another alkyl bromide group, new departure and finally ring closure (Figure 1).

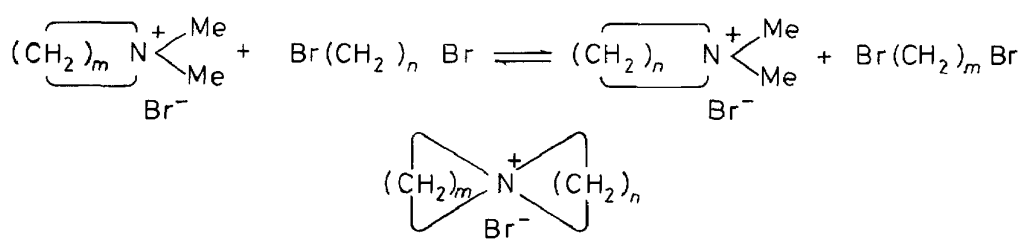

Figure 1

Mr Jostein Krane could, in fact, achieve isomerization at temperatures above $140^{\circ}$, but, unfortunately, so many other products were present in the mixture, notably of spiro-type (Figure 1), that the analytical problem became too formidable. This approach was therefore dropped, and quite lightheartedly, since it may be objected that the presence of geminal substituents on nitrogen must influence the relative stabilities of the various rings. This influence in itself presents, of course, another interesting conformational problem.

The presence of a single methyl group on nitrogen should be less objectionable since the $N$-methyl group is always able, by inversion, to accommodate itself in the least hindered position (e.g. equatorial in the six-membered ring) and will not perturb the ring conformation. Mr Krane's next attempt was therefore to establish the equilibrium between free tertiary amine bases through a reversible quaternization (Figure $2 a$ ). As the use of polymethylene dibromides $(\mathrm{X}=\mathrm{Br}$ ) would result in irreversible salt formation, $\mathrm{HX}$ must be a weaker acid than $\mathrm{HBr}$. If, on the other hand, $\mathrm{HX}$ is too weakly acidic, no catalytic formation of quaternary salts is possible. In practice, equilibration could be achieved between $150^{\circ}$ and $190^{\circ}$ by using certain substituted bisphenol ethers ( $\mathrm{X}=p$-nitrophenoxy), and a $\Delta H=-5.0 \mathrm{kcal} / \mathrm{mole}$ and a $\Delta S=-13$ e.u. was obtained for the thermodynamic equilibrium. For the equilibrium between the corresponding hydrocarbons (Figure $2 b$ ) thermochemical data lead to calculated values of $\Delta H=-6.0 \mathrm{kcal} / \mathrm{mole}$ and $\Delta S=$ -7.9 e.u. in qualitative agreement. Accepted ${ }^{2}$ average increments of $\Delta H^{0}$ 
and $S^{0}$ are similar for aliphatic and alicyclic $\mathrm{CH}_{2}$-groups, so that the negative $\Delta H$ reflects the smaller strain and the negative $\Delta S$ the lower entropy of the six-membered than of the five-membered ring. If it is assumed that the introduction of the hetero-atom does not change the ring conformations, and that the introduction of the X-group does not influence differently the conformational situation for aliphatic chains of different lengths, the effects of the hetero-atoms and the X-groups will cancel in the equilibrium. The smaller negative value observed for $\Delta H$ must then mean that the hetero-fivering is less strained than cyclopentane, possibly because the rotational barrier may be smaller in the CN-bond than in the CC-bond. On the other hand, the larger negative $\Delta S$ observed, if real, is then contrary to expectations, since pseudorotation in the five-membered ring would be impeded by a lower $\mathrm{CN}$ rotational barrier, and since the symmetry number of the sixmembered ring would be reduced by the hetero-atom. Solvation effects may be invoked, but their role is unknown.

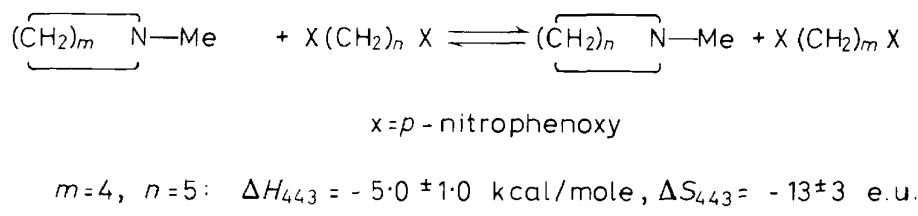

Figure $2 a$

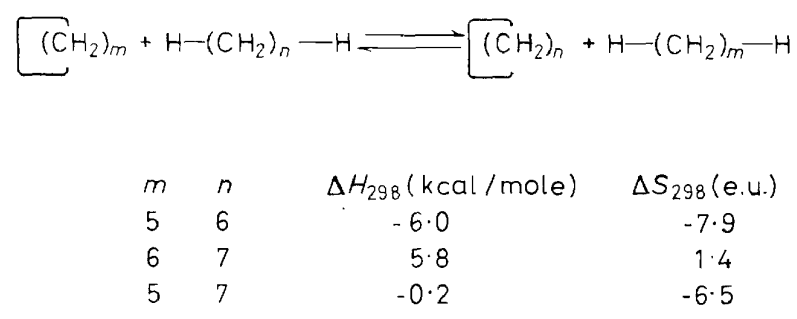

Figure $2 b$

Recently, Mr Tore Fagerhus showed that equilibrium determination over a wider temperature range was possible with cyclic ethers and polymethylene dibromides using $\mathrm{BF}_{3}$ as a catalyst at temperatures from $115^{\circ}$ to $180^{\circ} \mathrm{C}$ (Figure $3 a$ ). The hetero-atom is now oxygen and carries no ring substituent. so that this system should be a still simpler and cleaner case. Nevertheless, the data gave essentially the same values for 5-ring against 6-ring. Equilibration was also performed with 6-ring against 7-ring, and as a cross-check with 
5-ring against 7-ring (Figure 3a). The results are to be compared with calculated values for the corresponding hydrocarbon equilibria (Figure $2 b$ ).

Finally, equilibrium between cyclic diethers of polymethylene formal type could be established at even lower temperatures $\left(60^{\circ}-145^{\circ}\right)$ in the presence of acid catalysts (Figure $3 b$ ). Again, $\Delta H$ and $\Delta S$ turned out to have values very similar to those for the ring equilibria already discussed. Replacement of the formaldehyde component with acetaldehyde or acetone obviously permits the study of the relative influence of substituents on strain and pseudoration in the various ring sizes.

Such competition reactions can also be extended to medium and higher rings, but may then be complicated by formation of polymers and cyclic oligomers in addition to the two cyclic monomers.

(2). Recently, we have reported ${ }^{3}$ that cis,cis-cyclodeca-1,6-diene, and a series of its derivatives synthesized by Mr Terje Ekeland and carrying substituents in the 4- and 9-positions, have surprisingly high n.m.r.-coalescence temperatures for the geminally non-equivalent $\alpha-\mathrm{CH}_{2}$-protons. For two of the compounds $\mathrm{Mr}$ Jan Schaug determined the barrier height. One of these was independently determined by Servis and collaborators ${ }^{4}$, who also determined the barrier for the diketone, while $\mathrm{Lehn}^{4 a}$ has very recently determined the barrier height for three further derivatives (see Table 1). The results were interpreted in terms of inversion of a stable centrosymmetric 'arm-chair' conformation through a less stable 'hammock' conformation (Figure 4).

$$
\begin{array}{cccc}
\underbrace{\left(\mathrm{CH}_{2}\right)_{m} \mathrm{O}}_{m}+\mathrm{Br}\left(\mathrm{CH}_{2}\right)_{n} \mathrm{Br} \rightleftharpoons\left(\mathrm{CH}_{420}(\mathrm{kcal} / \text { mole })\right. & \Delta S_{420}(\mathrm{e} . \mathrm{u}) \\
4 & 5 & -5.0 \pm 0.5 & -12.8 \pm 1.2 \\
5 & 6 & 4.7 \pm 0.5 & 3.0 \pm 1.5 \\
4 & 6 & -1.9 \pm 1.0 & -9.1 \pm 2.0
\end{array}
$$

Figure $3 a$

$$
\begin{aligned}
& (\underbrace{\left.\mathrm{H}_{2}\right)_{m}}_{\mathrm{C}}{ }^{\mathrm{O}} \mathrm{CH}_{2}+\mathrm{HO}-\left(\mathrm{CH}_{2}\right)_{n}-\mathrm{OH} \rightleftharpoons(\underbrace{\left.\mathrm{H}_{2}\right)_{n}} \mathrm{CH}_{2}+\mathrm{HO}-\left(\mathrm{CH}_{2}\right)_{m}-\mathrm{OH} \\
& \begin{array}{ccrr}
m & n & \left.\Delta H_{375} \mathrm{kcal} / \text { mole }\right) & \Delta S_{375} \text { (e.u.) } \\
2 & 3 & -4.2 \pm 0.8 & -11.8 \pm 1.5 \\
3 & 4 & 4.0 \pm 0.5 & 2.8 \pm 1.0 \\
2 & 4 & -2.4 \pm 0.6 & -10.0 \pm 1.2
\end{array}
\end{aligned}
$$




\section{CONFORMATIONAL STUDIES OF SOME RING SYSTEMS}

Table 1. Inversion barriers for cyclodecadiene derivatives<smiles>[R]CC/C=C\CC=C</smiles>

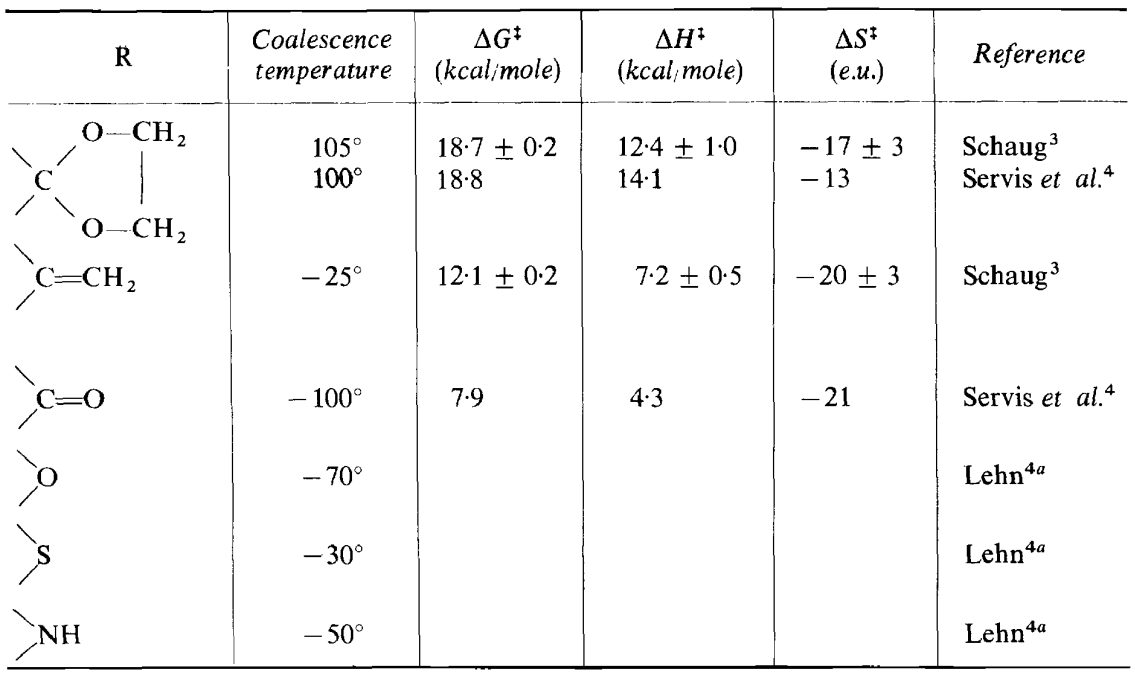

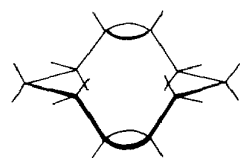

A

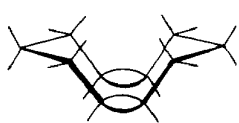

H

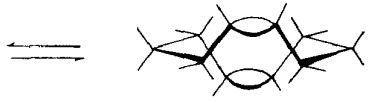

$A^{\prime}$

Figure 4

The observed barrier heights and their relative magnitudes are entirely reasonable for a true inversion process as far as the enthalpy of activation is concerned; the large negative value for the entropy of activation may reflect a high degree of coordination of atomic movements necessary to follow the lowest path over the barrier; that is, the transmission coefficient is very small. What really made the barrier seem surprising was the fact that no n.m.r.-coalescence phenomenon is observed for the most common tenmembered rings: cyclodecane and cyclodecanone. Several elements of 'flexibility' are introduced when going from cyclodecane to cyclodecadiene; 
wider ring angles, fewer hydrogen atoms, and lower torsional barriers next to the double bonds ${ }^{5}$. It is therefore hard to accept that the ring inversion barrier should be higher in the unsaturated compound. It suffices to recall that cyclohexene has a barrier height ${ }^{6}$ of $5 \cdot 3 \mathrm{kcal} /$ mole $\left(\Delta H^{\ddagger} \approx \Delta G^{\ddagger}\right)$, much lower than that of $10.8 \mathrm{kcal} / \mathrm{mole}\left(\Delta H^{\ddagger}\right)$ for cyclohexane ${ }^{7}$, and that olefins have smaller activation energies for viscous flow than paraffins ${ }^{8}$. It is more likely that a genuine inversion process through partial ring flattening and bond-angle opening has a relatively high barrier also in cyclodecane and cyclodecanone, but that exchange of substituent sites proceeds over low* energy 'pseudorotation' barriers'. 'Pseudorotation' is here used in the rather broad sense of asymmetrical modes of conformational transformations involving mainly passage over torsional barriers and little bond-angle opening or compression of non-bonded atoms. Since $\mathrm{CH}_{2}$ and $\mathrm{C}=\mathrm{O}$ groups may occupy any position on the skeleton of the preferred conformation of cyclodecane ${ }^{10}$, each of these can wander around the ring by successive steps without hindrance. In cis,cis-cyclodeca-1,6-diene ${ }^{3}$ there are three types of ring atoms that cannot exchange sites, and pseudorotation becomes blocked. There remains then only a genuine inversion path through two or several steps of the type proposed in Figure 4 to effect site exchange on the $\alpha$-carbon atoms.

This viewpoint has recently been supported by the observation that some bis-geminal-dimethyl-substituted medium-ring ketones, obtained by Mrs Gerd Borgen as byproducts in the synthesis of macrocyclic diketones (to be described in the next Section), displayed n.m.r. coalescence. As just pointed out, the corresponding unsubstituted ketones show no sign of coalescence, and it is difficult to see how gem-dimethyl groups could hinder ring inversion via a ring flattening/bond-angle opening mechanism. Thus, the inversion barrier of 1,1-dimethylcyclohexane ${ }^{11}\left(\Delta G^{\ddagger}=10 \cdot 4 \mathrm{kcal} / \mathrm{mole}\right)$ is essentially the same as that of cyclohexane itself $^{7}\left(\Delta G^{\ddagger}=10 \cdot 3 \mathrm{kcal} / \mathrm{mole}\right)$. On the other hand, since the favoured conformations for medium-sized cycloalkanes, and presumably also for the ketones, permit gem-dimethyl groups to occupy only certain ring-positions, the pseudorotation process responsible for the rapid site exchange of geminal substituents would be thereby impeded and only a slower inversion process remain available.

In the synthesis of these monoketones by Dieckmann cyclization ${ }^{12}$ of tetramethyl-substituted diesters, the yields alternate in a striking manner with ring size (Table 2). On the basis of the well-established cyclization promoting 'gem-dimethyl effect' one might have expected that the yield of cyclic monoketones, as compared with the corresponding unsubstituted compounds, should increase quite generally, and that the ratio of cyclic mono- to cyclic di-ketone should also increase. The data of Table 2 show that this is only the case when the ring is 9- to 12 -membered, and even here to a varying degree. It is most pronounced when the two gem-dimethyl substituted atoms are separated by two $\mathrm{CH}_{2}$-groups and less pronounced when they are separated by three $\mathrm{CH}_{2}$-groups. For the 7- and 8-membered ring monoketones an apparent negative gem-dimethyl effect is observed. However, in these cases the reactivity of the starting diester is so low that most of it was recovered and no cyclic diketone or polymer could be isolated. The reason is probably of a steric nature as the $\alpha-\mathrm{CH}_{2}$ group which is to be 
Table 2. Cyclization of diesters

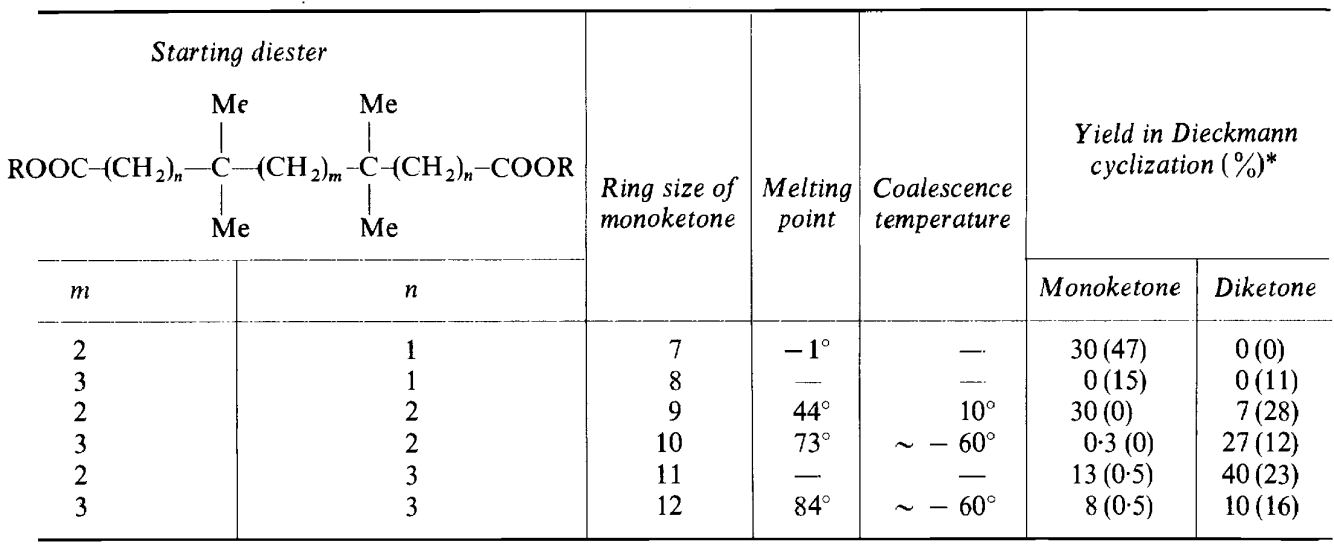

Yield of corresponding unsubstituted compound in parenthesis ${ }^{12}$.

attacked by the base is too close to the dimethyl-branching. On this basis, one may even guess that the pattern from the higher members extends down the series. That only the cycloheptane derivative with two $\mathrm{CH}_{2}$-groups between the branching points has been isolated, and not the cyclo-octane derivative having three, supports this.

An explanation of the observed difference in cyclization-promoting effect of two gem-dimethyl groups in 1,4- and 1,5-position can be arrived at by considering the conformational possibilities of the diester chain. Cyclization to rings which are 9-membered or smaller is assumed to require a sequence of only gauche-bonds, while for cyclization to the larger of these rings separate anti-bonds can also be accepted, but not an uninterrupted sequence of two or more anti-bonds. The chain CC-bonds immediately adjoining the gem-dimethyl-substituted carbon atoms have twice as high a probability of being gauche as anti since no enthalpy difference is expected. In the 1,4 case (Figure 5) there is one $\mathrm{CH}_{2}-\mathrm{CH}_{2}$ bond in the middle, and both the lower energy anti-form and the higher energy gauche-form can lead to cyclization, the former being restricted to 10 -membered and higher rings.

In the 1,5 case (Figure 6), on the other hand, there are two $\mathrm{CH}_{2}-\mathrm{CH}_{2}$ bonds in the middle of the chain, and the lowest energy form, anti, anti, will not here permit cyclization except possibly to the 12-membered ring. The next lowest energy form, gauche, anti, will permit cyclization only to 10 membered and higher rings. The probability of having two gauche-bonds, and more specifically of the chirality sequence required for cyclization, is very much lower.

The melting points and the n.m.r.-spectra at varying temperatures (Table 2) suggest that among these medium-ring ketones, the 9-membered, and perhaps the 10 - and 12 -membered, are rigid and conformationally homogeneous. Thus, the melting points are particularly high for these rings, and a coalescence phenomenon is observed for the 9 -ring ketone at $\sim 10^{\circ} \mathrm{C}$, whereby the single high-temperature $\mathrm{CH}_{3}$-signal is split into a low-temperature doublet as ring inversion becomes sufficiently slow. The activation 


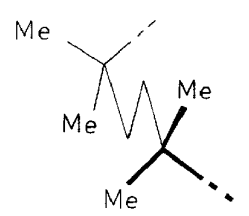

$+g, \underline{a},+g$

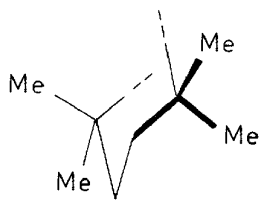

$+g,-\underline{g},+g$

Figure 5

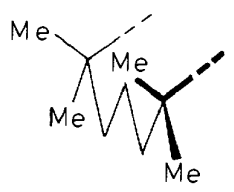

$-g, a, a,+g$

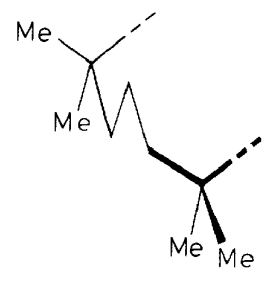

$+g,+g, a,+g$

Fiqure 6

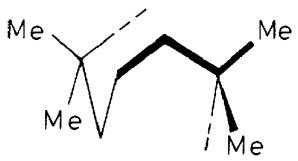

$+g,-g,-g,+g$

energy for this process has been determined by Mr Schaug to be surprisingly high; $\Delta G^{\ddagger}=15.2 \pm 0.2 \mathrm{kcal} / \mathrm{mole}, \Delta H^{\ddagger}=14 \pm 2 \mathrm{kcal} / \mathrm{mole}, \Delta S^{\ddagger} \sim 0$. For the 10- and 12-ring ketone the coalescence temperatures seem to be just below $-60^{\circ}$; for the other rings no broadening is observed down to $-60^{\circ}$.

The unique conformation of 4,4,7,7-tetramethylcyclononanone must have two equivalent gem-dimethyl groups, and within each the two methyl groups must occupy non-equivalent sites. For symmetry reasons this suggests that the naked 9-ring skeleton has the $D_{3}$ symmetry calculated by Hendrickson ${ }^{13}$ to be the lowest-energy conformation for cyclononane and that both gemdimethyl groups as well as the carbonyl group occupy the positions which had $C_{2}$ symmetry in the unsubstituted skeleton (Figure 7).

4,4,8,8-Tetramethylcyclodecanone is unable to adopt the well-established stable cyclodecane ring skeleton, as two gem-dimethyl groups in these relative positions cannot both occupy allowed corner positions (Figure 8). Alternative but more strained skeletons for a similar tetramethyl-substituted decane have been found with x-ray methods by Dunitz and $\operatorname{Eser}^{10}$ (Figure 8). We suggest that the least strained of these conformations is also the one adopted by our ketone. This is to some extent confirmed by the similar n.m.r. signals for all three $\mathrm{CH}_{2}$-groups $\left(\mathrm{C}_{5}-\mathrm{C}_{7}\right)$. suggesting that the carbonyl group cannot be close enough in space to influence their chemical shift. 


\section{CONFORMATIONAL STUDIES OF SOME RING SYSTEMS}

It is perhaps not surprising that 5,5,8,8-tetramethylcycloundecanone is conformationally less well-defined. This is not necessarily a result of an awkward relative position of the methyl groups, but rather inherent in the odd-membered ring skeleton ${ }^{1}$.

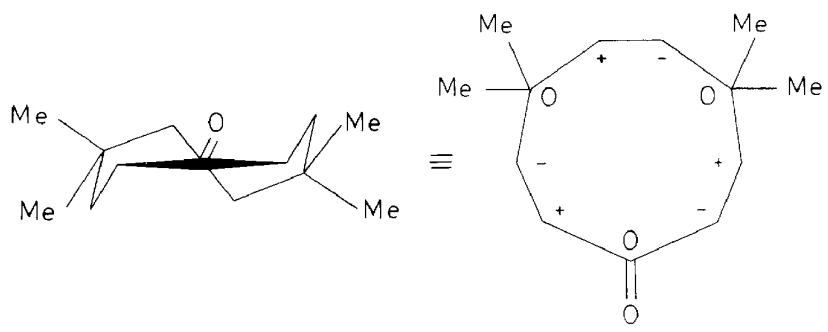

Figure 7
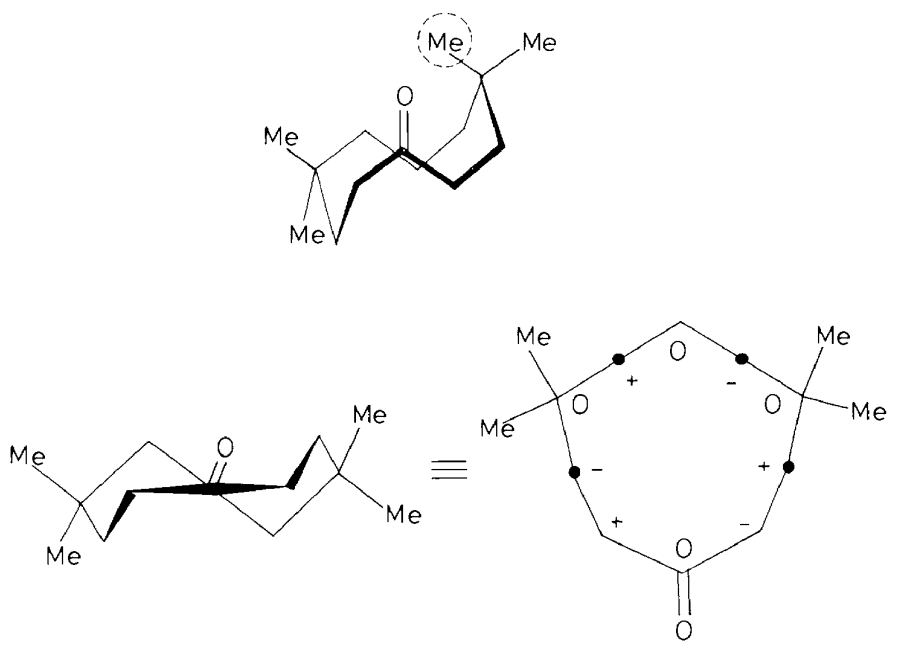

Figure 8

For 5,5,9,9-tetramethylcyclododecanone the observed high conformational stability is not compatible with the square 'non-diamond lattice' conformation established ${ }^{14}$ for cyclododecane itself and certain derivatives, since the gem-dimethyl groups cannot both be in the sterically allowed corner positions (Figure 9). There are, however, two possible 'diamond lattice' conformations for the skeleton, a 'triangular' one and a 'rectangular' one (Figure 9), both of which can accommodate the gem-dimethyl groups in corner positions. The former has one serious triple-hydrogen interaction, while the latter has two less serious hydrogen-hydrogen interactions (three for the parent 
hydrocarbon). The latter may therefore be favoured. This is supported by observed chemical shifts of the $\mathrm{CH}_{2}$-groups in positions 6-8 which are no longer coincident (as in the comparable tetramethylcyclodecanone above). The carbonyl group of the latter conformation would clearly come closer to one of these $\mathrm{CH}_{2}$-groups than in the former.
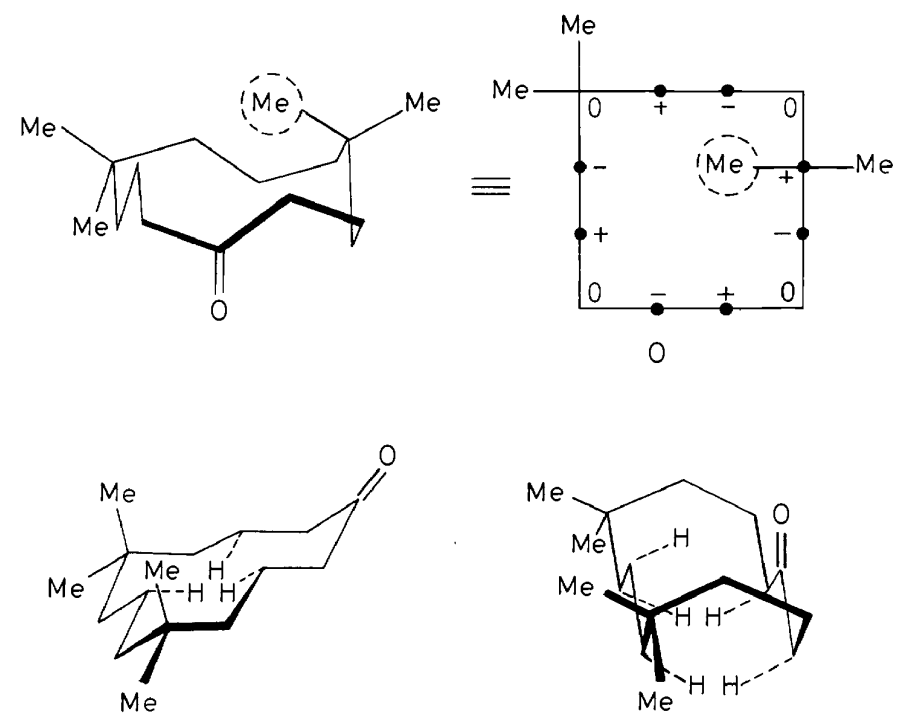

Figure 9

(3). The Dieckmann cyclization of diesters just described also gave the desired cyclic diketones of twice the ring size (Table 2), except the 14- and 16-membered ring. Starting material was largely recovered, so that it is clear that the main reason is a low reactivity, presumably due to the proximity of the dimethyl branching, which hinders either the attack on the $\alpha-\mathrm{CH}_{2}$ group by the base or, more likely, attack by the formed bulky anion on the carbonyl of another ester group. It was therefore expected that the Blomquist ketene cyclization $^{15}$ would be more successful, as both the ketene group and its 'dimerization' product, the $\beta$-lactone group, would be relatively less bulky, and the reaction itself more rapid. This proved to be the case, and Mrs Borgen obtained both the 14- and 16-membered cyclic diketone in moderate yield.

For the type of conformational studies we had in mind and which were concerned with the effect of dimethyl groups on the hydrocarbons, the presence of the keto-groups in these macrocyclic diketones presents certain complications. Thus, the small rotational barriers in the CC-bonds adjoining the carbonyl groups ${ }^{5}$ introduces a flexibility at these points, and a deviation from perfect diamond-lattice geometry becomes easier, for example as a result of dipole-interaction between the carbonyl groups. On the other hand, the observed molecular dipole moments might give information about the 
relative orientation of the two carbonyl groups. These compounds were therefore examined by several methods.

The expected most stable conformations for the parent cycloalkanes are rectangular in shape with the gem-dimethyl groups in corner positions ${ }^{1}$. If these are also adopted by the cycloalkanediones, their best conformations should be as shown in Figure 10. It can be seen that in the two-carbonbridged series (14-, 18-, 22-membered rings) the carbonyl dipoles are antiparallel, whereas in the three-carbon-bridged series (16-, 20-, 24-membered
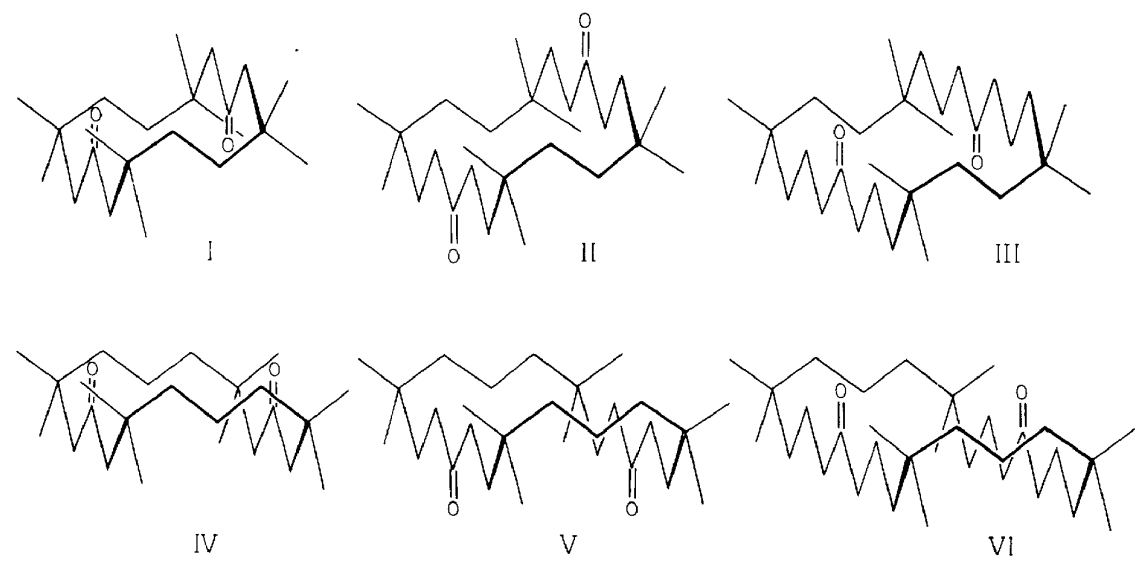

Figure 10

rings) they are parallel. The observed dipole moments (Table 3) might seem strikingly to confirm this prediction as the former series have dipole moments much smaller than those of the latter series. The values observed lie respectively below and above the value of $2.7 \mathrm{D} \times \sqrt{2}=3.8 \mathrm{D}$ calculated ${ }^{16}$ for free relative orientation. However, for some of these rings there are

Table 3. Properties of octamethylcycloalkanediones

\begin{tabular}{c|c|c|c|c|c|c|c}
\hline $\begin{array}{c}\text { Cyclic } \\
\text { diketone } \\
\text { (Figure 10) }\end{array}$ & $\begin{array}{c}\text { Number } \\
\text { of ring } \\
\text { atoms }\end{array}$ & $\begin{array}{c}\text { Melting } \\
\text { point }\end{array}$ & $\begin{array}{c}\Delta H_{m} \\
(k \text { kcal/mole })\end{array}$ & $\begin{array}{c}\Delta S_{m} \\
(e . u .)\end{array}$ & $\begin{array}{c}\text { Dipole } \\
\text { moment } \\
\text { (Debye) }\end{array}$ & $\begin{array}{c}\text { Solubility } \\
\text { in } \text { CS }_{2}\end{array}$ & $\begin{array}{c}\text { Infra-red spectrum } \\
\text { in solution } \\
\text { as compared } \\
\text { to solid }\end{array}$ \\
\hline I & 14 & $219^{\circ}$ & $5 \cdot 9$ & $12 \cdot \hat{2}$ & $1 \cdot 1$ & slight & unaltered \\
IV & 16 & $150^{\circ}$ & $8 \cdot 2$ & $19 \cdot 5$ & 4.4 & good & unaltered \\
II & 18 & $220^{\circ}$ & $12 \cdot 1$ & 24.5 & 1.7 & very slight \\
new bands \\
V
\end{tabular}




\section{J. DALE}

alternative conformations with the same relative dipole orientation. Strictly, therefore, it can only be said that the observed dipole moments are not in disagreement with the conformations shown in Figure 10.

No such alternative hydrocarbon conformations with only staggered bonds (corresponding to cyclic paths on the diamond lattice) and closest admitted non-bonded $\mathrm{H}-\mathrm{H}$ interactions as in gauche-butane exist for the 14- and 16-membered rings ${ }^{1}$, not even when substituents are lacking, but several are possible for the higher members of both series. For the nextlowest member of each series, all possibilities are shown in the next two figures; for cyclo-octadecane there are four (Figure 11), and for cycloeicosane five (Figure 12).
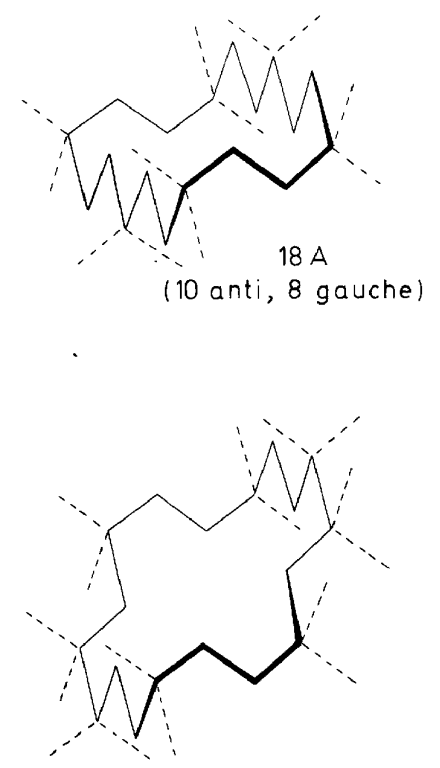

$18 \mathrm{C}$

(10 anti, 8 gauche)

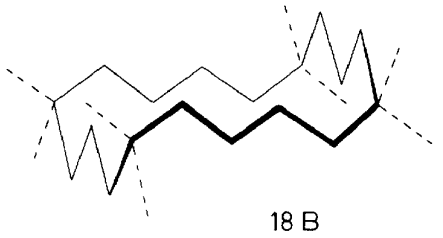

(10 anti, 8 gauche)

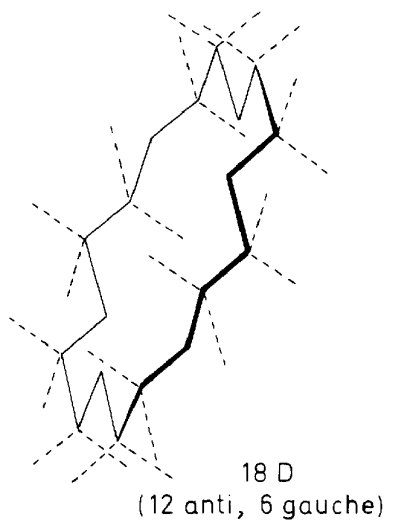

(12 anti, 6 gauche)

Figure 11

The unsubstituted 18-membered ring will have a maximum number of low-energy anti-bonds in conformation $18 \mathrm{D}$ of Figure 11, and this may well be dominant, but not exclusive, in the liquid and solution. Conformation $18 \mathrm{~A}$ is, however, more compact and very probably the one present in the low-temperature crystal lattice (cf. cyclotetratriacontane ${ }^{17}$ ). When dimethyl groups are introduced as by our synthesis ${ }^{18}$, in relative positions $1,4,10$, and 13, it is possible to fit all of them into allowed positions (indicated by dotted substituent lines) of conformations $18 \mathrm{~A}, \mathrm{C}$ and D. However, only in conformation $18 \mathrm{~A}$ can they be placed at corners so that they can be adjoined by all eight gauche-bonds and effectively cancel here the energy difference 


\section{CONFORMATIONAL STUDIES OF SOME RING SYSTEMS}

between gauche- and anti-bonds. Only four of the six gauche-bonds in conformation $18 \mathrm{D}$, can, for example, be similarly improved. When carbonyl groups are also present (in positions 7 and 16) the situation is not so clear, since it is presumably less important for the $\mathrm{CC}$ bonds adjoining the carbonyl group to maintain an anti-orientation than it is for the $\mathrm{CH}_{2}-\mathrm{CH}_{2}$ bonds of of the hydrocarbon. Conformations $18 \mathrm{D}$ and even $18 \mathrm{C}$ may then again become serious competitors. All these conformations have centres of symmetry, and diametrically placed keto groups must therefore always cancel the dipole moment of each other.

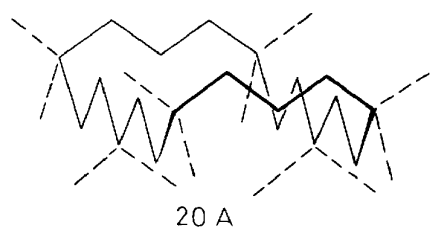

(12 anti, 8 gauche)

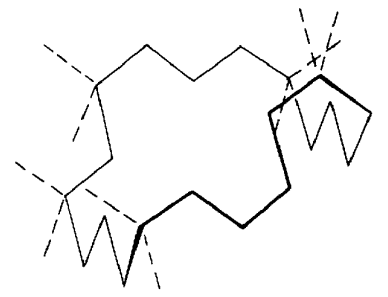

$20 \mathrm{~B}$

(10 anti, 10 gauche)

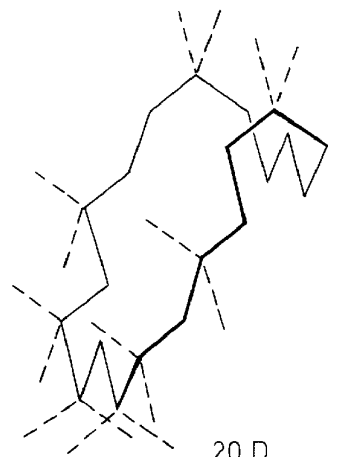

(10 anti, 10 gauche)

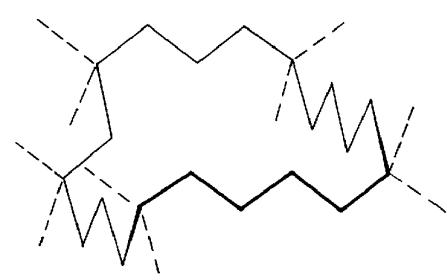

$20 \mathrm{C}$

(12 anti, 8 gauche)

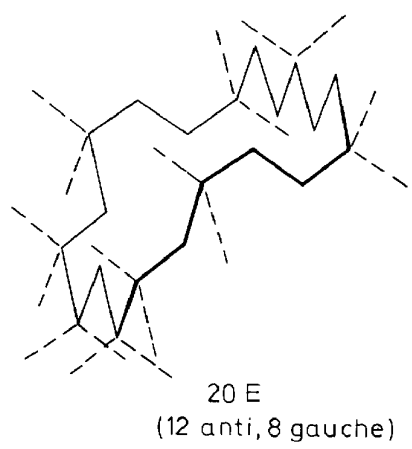

Figure 12 


\section{J. DALE}

The unsubstituted 20 -membered ring will have a maximum number of low-energy anti-bonds in conformations $20 \mathrm{~A}, \mathrm{C}$ and $\mathrm{E}$ of Figure 12 . None of these are compact and it is not known which is present in the crystal. When dimethyl groups occupy relative positions $1,5,11$, and 15 , as in our synthesis, conformation $20 \mathrm{~A}$ is the only one that can admit them without steric hindrance, and the reduction of gauche-bond energy directs them towards corner positions. Carbonyl groups in positions 8 and 18 should then end up in the middle of the 'long sides' and be oriented parallel so that their dipole moments will add.

For the 22- and 24-membered rings a still greater number of diamondlattice conformations are possible.

Amongst other properties given in Table 3 the solubilities in $\mathrm{CS}_{2}$ and the melting points belong to the same category as the dipole moments. The two-carbon-bridged rings have a centre of symmetry, are more compact, and can more efficiently give intermolecular dipole interaction than the three-carbon-bridged rings (cf. macrocyclic diamides ${ }^{19}$ ). They would therefore have the higher melting points and the lower solubilities, as was indeed observed. When the melting point is examined more closely by determination of enthalpy and entropy of melting, however, this is not quite so simple. Only if the 14-membered ring is excluded for a moment from the comparison, do the data make sense. Not only the higher enthalpies of melting but also the higher entropies of melting belong then to the centrosymmetric rings. This can only mean that for the 18- and 22-membered ring, alternative conformations of the type already discussed are indeed present in the melt, while the 16-, 20- and 24-membered rings have to a greater extent become locked up in one conformation by the gem-dimethyl groups. Returning now to the 14-membered ring, its small enthalpy of melting may reflect a steric hindrance to intermolecular dipole interaction, and its small entropy of fusion that only a single conformation is possible.

More direct information as to conformational homogeneity is obtainable from a comparison of the infra-red spectra in the crystalline state and in solution. The results (Table 3) show that no new bands appear in solution for the 14- and 16-membered rings, so that these are clearly conformationally homogeneous. The higher rings do show new bands in solution, and for the 18- and 22-membered rings these are probably due to the alternative types shown in Figure 11. What the alternative conformations can be for the 20- and 24-membered rings is not clear, since it has just been shown that for the former only the rectangular ring conformation $20 \mathrm{~A}$ is strain-free. Possibly, non-diamond lattice conformations with partially eclipsed bonds next to the carbonyl groups, advantageous entropy-wise, are present.

Low-temperature n.m.r. spectroscopy shows an initial methyl line broadening at $-60^{\circ}$ for the same two smallest rings that are also conformationally homogeneous. At higher temperature these show one sharp methyl signal, as do all the higher members.

The corresponding gem-dimethyl substituted hydrocarbons were expected to be much more well-defined conformationally than the cyclic diketones. Transformation of these to the saturated hydrocarbons was attempted using the standard Wolff-Kishner reduction method ${ }^{20}$. For the 18-membered and higher rings this presented no problem, except that a 


\section{CONFORMATIONAL STUDIES OF SOME RING SYSTEMS}

rather long reaction time was needed. The 14- and 16-membered rings, however, strongly resisted reduction, presumably because of steric hindrance from the gem-dimethyl groups, which are here closer to the carbonyl group than in the higher homologues. Just these two rings were also difficult to obtain by cyclization.

The pure cycloalkanes with 18-, 20-, and 22-membered rings obtained have the properties shown in Table 4. It is of particular interest that, contrary to the situation for the parent diketones, the infra-red spectra of the solid and of solutions in $\mathrm{CS}_{2}$ are nearly identical, proving that hardly any new conformations are present in solution. It is of equal interest that initial broadening of the n.m.r.-lines is observed on cooling, suggesting hindered ring inversion. One therefore has good reason to believe that at room temperature all three cycloalkanes are present predominantly in the rectangular conformations of the type $18 \mathrm{~A}$ and $20 \mathrm{~A}$ shown in Figures 11 and 12 , and with all eight methyl groups in corner positions. Although not yet isolated, the cycloalkanes with 14 and 16 ring members are expected to behave similarly, as their parent diketones are already conformationally homogeneous.

The calorimetric data for the melting process (Table 4) are more difficult

Table 4. Properties of octamethylcycloalkanes

\begin{tabular}{c|c|c|c|c|c|c}
\hline $\begin{array}{c}\text { Parent } \\
\text { diketone } \\
\text { Figure 10 }\end{array}$ & $\begin{array}{c}\text { Number } \\
\text { of ring } \\
\text { atoms }\end{array}$ & $\begin{array}{c}\text { Melting } \\
\text { point }\end{array}$ & $\begin{array}{c}\Delta H_{m} \\
(\mathrm{kcal} / \text { mole })\end{array}$ & $\begin{array}{c}\Delta S_{m} \\
(e . u .)\end{array}$ & $\begin{array}{c}\text { Beginning } \\
\text { broadening } \\
\text { in n.m.r. }\end{array}$ & $\begin{array}{c}\text { Infra-red spectrum } \\
\text { in solution as } \\
\text { compared to solid }\end{array}$ \\
\hline II & 18 & $164^{\circ}$ & $6 \cdot 1^{*}$ & $13 \cdot 8^{*}$ & $-30^{\circ}$ & no new bands \\
V & 20 & $166^{\circ}$ & $11 \cdot 4$ & $26 \cdot 4$ & $-50^{\circ}$ & only slight changes \\
III & 22 & $138^{\circ}$ & $14 \cdot 0$ & $34 \cdot 1$ & $-60^{\circ}$ & only slight changes \\
\hline
\end{tabular}

* Total melting. Part of the melting process takes place at a transition point at $155^{\circ}\left(\Delta H_{\mathrm{tr}}=1.4 \mathrm{kcal} / \mathrm{mole} . \Delta S_{1 \mathrm{r}}=5 \cdot 2 \mathrm{e} . \mathrm{u}.\right)$

to understand. The values for the 18-membered ring are appropriate for a rigid molecule of this symmetry, but for the two higher rings both the enthalpy and entropy of melting seem too high. One possible explanation for the high entropy is that alternative conformations may be much more heavily populated in the melt at about $140^{\circ}$ to $170^{\circ}$ than in solution at $20^{\circ}$.

A marked tendency for crystals of the 20 -membered cycloalkane, but not for the others, to retain solvent molecules (methanol, $\mathrm{CCl}_{4}$ ) may be due to the need to fill the void of the open three-carbon-bridged conformation $20 \mathrm{~A}$.

The above results point to the possibility of using the octamethylcyclotetradecane and -cyclohexadecane skeletons as playing-fields for conformational studies similar in scope to the field of cyclohexane chemistry. Since these molecules have a rigid skeleton, an additional substituent, as long as it is constitutionally defined, should also be conformationally defined, except as to its being external or internal (in cyclohexane: equatorial or axial). However, a severe limitation has already been indicated; the steric hindrance of the gem-dimethyl groups to chemical transformations elsewhere in the molecule.

(4). The preceding Sections have dealt with small, medium and large rings 
separately. We now proceed to discuss two families of ring compounds, each of which includes chemically related rings of different sizes.

The first family (Figure 13) is a series of cyclic oligopeptides of sarcosine ( $N$-methylglycine) which Miss Kirsten Titlestad has recently succeeded in synthesizing ${ }^{21}$.

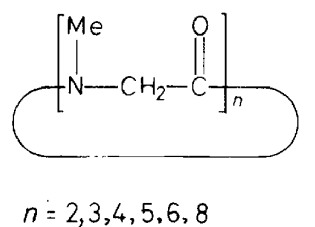

Figure 13

There has been much speculation about the role of transannular hydrogen bonding in stabilizing particular conlormations of cyclic peptides ${ }^{1}$. Our approach was to exclude hydrogen bonding by using the simplest $N$-methylated $\alpha$-aminoacid. These cyclic oligomers should then reveal more clearly the unperturbed ring conformation. A higher lipophilicity should also make dipole moment measurements and spectral studies in solution easier. On the other hand, due to the similarity between $-\mathrm{CH}_{3}$ and $-\mathrm{CH}_{2}-$, the strong trans-preference for the amide group should disappear on $N$-methylation. That is why the cyclic tripeptide, hitherto only reported ${ }^{22}$ for the rather special five-ring aminoacid proline, was formed easily. The free competition between the cis-and trans-amide configuration in rings less constrained than the cyclic dipeptide might yield information about the intrinsic conformation of the cis- and trans-amide groups.

To our great surprise it turned out that cyclo-tri-, tetra-, penta- and octasarcosyl, which are 9-, 12-,15- and 24-membered rings respectively, were both configurationally (cis, trans) and conformationally homogeneous. The evidence came mainly from the temperature-dependent n.m.r. spectra where coalescence phenomena occurred at relatively high temperatures. This is in striking contrast to the simple temperature-independent n.m.r. spectra of, for example, cyclo-tetra- and hexaglycyl, where hydrogen-bonding is possible and temperature dependence would a priori seem more likely. We are therefore forced to conclude that a stronger transannular attraction exists between $\mathrm{N}$-methyl amide groups than between $\mathrm{N}$-hydrogen amide groups. Before discussing the nature of this interaction, we shall briefly quote the exceptional amount of information which can be extracted from the n.m.r. spectra of these rings.

Least information was obtained for cyclodisarcosyl, whose simple two-line spectrum and lack of coalescence phenomenon indicates either a planar or rapidly inverting non-planar conformation. A slightly non-planar boat-like conformation is suggested by the dipole moment of $1 \cdot 13 \mathrm{D}$ observed in 
benzene solution, but the crystal structure determined by Mr Per Groth reveals an extremely flattened chair form with a centre of symmetry. Each amide group contains the $\mathrm{N}$-methyl carbon, but not its $\mathrm{N}-\mathrm{CH}_{2}$ carbon, in the common plane (dihedral angle CCNC $=7^{\circ}$ ).

For cyclotrisarcosyl the presence (Figure 14) of one singlet for $\mathrm{N}$-methyl and one quartet for $\mathrm{CH}_{2}$, intensity $9: 6$, shows that all amide groups have the same configuration, and since they cannot all be trans in a nine-membered ring, they must all be cis. The very large splitting of the $\mathrm{CH}_{2}$ signals means that the ring is far from planar, and the high coalescence temperature of around $135^{\circ}$ that it is very rigid. The only conformation that satisfies these data is that of Figure 15 with all carbonyl groups on the same side. It is to some extent confirmed by the high dipole moment of $4.66 \mathrm{D}$ observed in benzene solution.

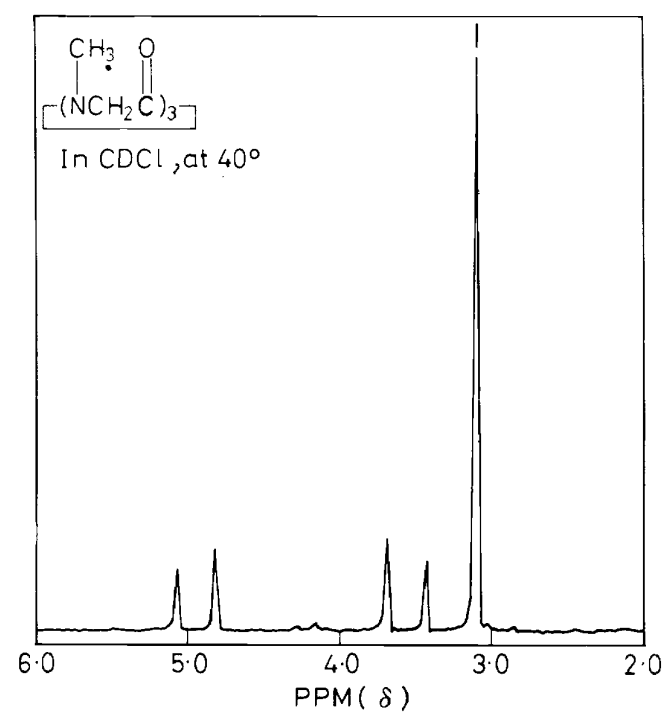

Figure 14

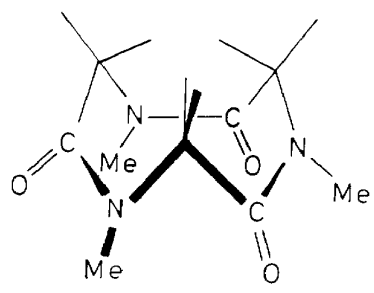

Figure 15 


\section{J. DALE}

Cyclotetrasarcosyl shows two $N$-methyl signals (Figure 16), intensity $6: 6$, and two $\mathrm{CH}_{2}$ quartets, intensity $4: 4$, which means that out of the six possible isomers there can be only one present. For symmetry reasons the configuration of the four amide groups must have the sequence cis, trans, cis, trans and the molecular conformation must be centrosymmetric. All lines start to broaden at $155^{\circ}$ and end up as two single lines $\left(\mathrm{CH}_{2}\right.$ and $\left.\mathrm{NCH}_{3}\right)$ at about $200^{\circ}$. Apparently, the two coalescence phenomena expected, collapse of each quartet into one line due to rapid ring inversion, and the further collapse of the two $\mathrm{CH}_{2}$ lines, as well as the two $\mathrm{N}$-methyl signals, into one line due to rapid interchange of amide configuration, occur in the same temperature range. The high temperature needed for coalescence to occur shows that inversion of this non-planar twelve-membered ring is remarkably difficult. Together with the configurational homogeneity, this suggests strong transannular attractions between at least two amide groups. Since hydrogen bonding is excluded, we proposed ${ }^{21}$ that contributions of transannular polar resonance structures (Figure 17) have to be considered as an alternative to the common polar resonance structures<smiles>CC([O-])=[N+](C)C</smiles>

The case is reminiscent of the transannular interaction observed by Leonard ${ }^{23}$ between an amino- and a keto-group in certain medium rings. An unusually short transannular contact between the ester oxygen and the ketone carbon

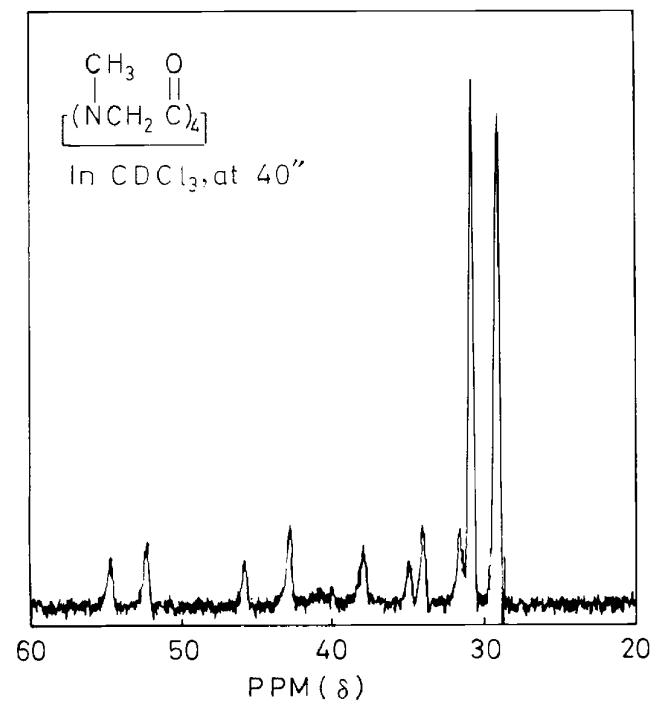

Figure 16 
has been found ${ }^{24}$ in the related ten-membered-ring lactone of 9-hydroxy-6oxo-nonanoic acid.

$\mathrm{Mr}$ Groth has now also solved the crystal structure of cyclotetrasarcosyl and finds, in fact, a molecule which has all the above features, including alternating cis-, trans-amide groups and the shortest transannular distances between nitrogen and carbonyl carbon of the trans-amide groups. A pyramidal configuration at carbonyl carbon, the apex pointing inwards, gives additional evidence of transannular interaction. Although the crystal structure does confirm a centre of symmetry, some details are different (Figure 18). It amounts really to a difference in expected and observed

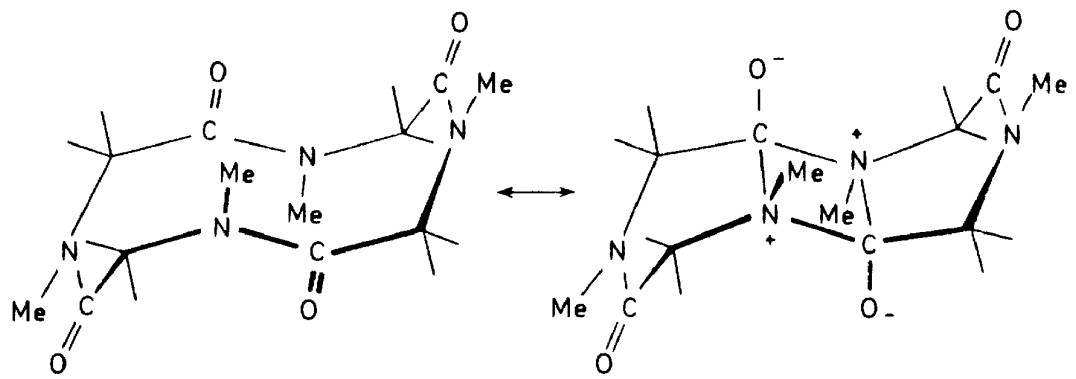

Figure 17

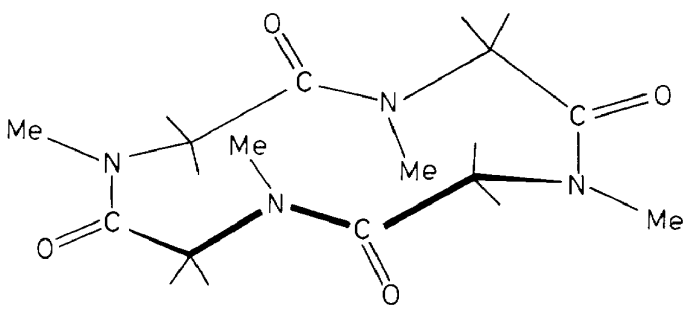

Figure 18

dihedral angles in the single bonds adjoining the amide groups. An interesting dualism is observed: on the one hand, the skeletal dihedral angle on the carbonyl side is close to staggered, $66^{\circ}$ and $170^{\circ}$ for cis and trans respectively, as in ketones, etc. ${ }^{5}$, and suggests a pure classical structure for the amide group. On the other hand, the skeletal dihedral angle on the nitrogen side is $121^{\circ}$ for trans, close to the favoured value for olefins ${ }^{5}$, suggesting a full $\mathrm{CN}$-double bond of a charged resonance structure, and an intermediate 
value of about $94^{\circ}$ for cis. These erring values may indicate that the $\mathrm{CN}$ barrier is actually quite low, possibly because of the fractional positive charge on nitrogen ${ }^{5}$.

Cyclopentasarcosyl (Figure 19) shows five $\mathrm{N}$-methyl lines, intensity $3: 3: 3: 3: 3$, and five recognizable $\mathrm{CH}_{2}$ quartets, intensity $2: 2: 2: 2: 2$. The observed integral intensity ratio means that even this fifteen-membered ring is present as one largely predominant isomer out of the eight possible ones, but symmetry arguments can only decide that it is not the all-cis or the all-trans isomer. Coalescence of the five $\mathrm{CH}_{2}$-quartets into five singlets, as ring inversion becomes fast enough, starts at about $40^{\circ}$ but is not finished before the $\mathrm{N}$-methyl lines also start to broaden at $70^{\circ}$, as configuration interchange becomes fast enough. At $130^{\circ}$ only two singlets $\left(\mathrm{CH}_{2}\right.$ and $\left.\mathrm{NCH}_{3}\right)$ are present. Again, the high resistance to ring inversion and the configurational and conformational homogeneity suggest strong transannular interactions between amide groups.

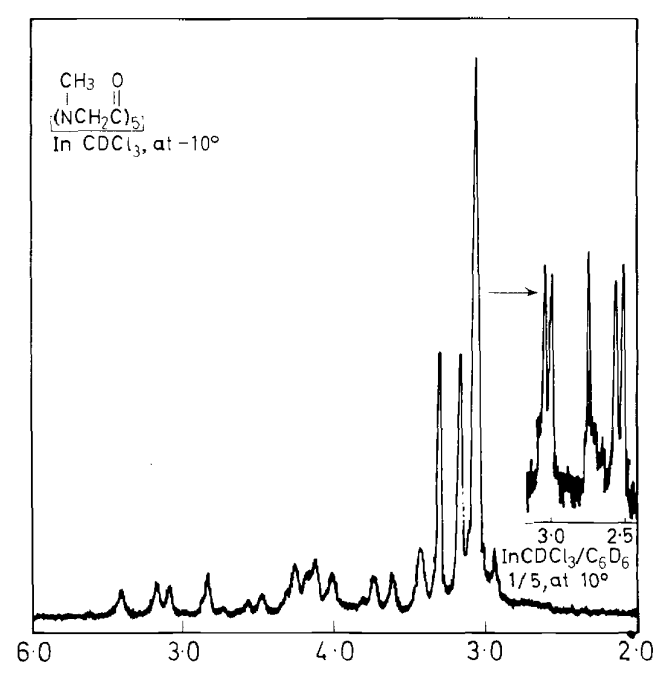

Figure 19

Cyclohexasarcosyl, on the other hand, has an n.m.r. spectrum from which no certain conclusions can be drawn concerning configuration sequence or even the number of isomers present (fourteen are possible here).

Cyclo-octasarcosyl finally shows (Figure 20) four $N$-methyl signals, intensity $6: 6: 6: 6$, more or less resolved in various solvent systems. At about $100^{\circ}$ coalescence into one $\mathrm{N}-\mathrm{CH}_{3}$ line (as well as into one $\mathrm{CH}_{2}$ line) is observed. By symmetry arguments, an integral intensity ratio of only four $\mathrm{N}-\mathrm{CH}_{3}$ lines would indicate that a single isomer out of the thirty-eight possible ones is present, that its configuration sequence is cis, cis, trans, trans, cis, cis, trans, trans, and that its conformation has a centre of symmetry. 


\section{CONFORMATIONAL STUDIES OF SOME RING SYSTEMS}

From the $\mathrm{CH}_{2}$ signals the conclusions are less clear, but not in contradiction. The overlapping unresolved quartets coalesce at $20^{\circ}$ to $40^{\circ}$ into two broad lines. It is clear that ring inversion is slow even for this twenty-four-membered macrocycle, and postulation of transannular interactions again seems unavoidable. The implications that this type of interaction between amide groups has for protein chemistry are self-evident.

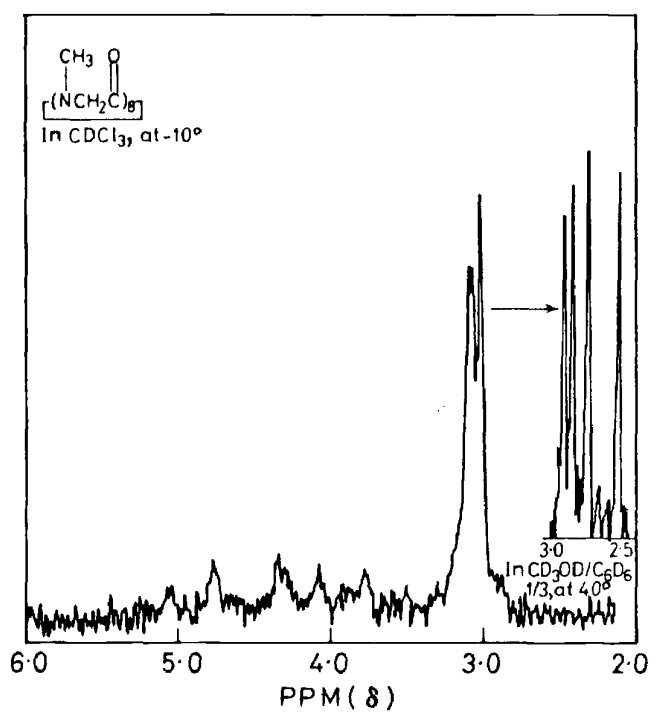

Figure 20

(5). The other family is a long series (Figure 21) of cyclic oligo(dimethylsiloxanes) which $\mathrm{Mr}$ Thor Alvik has isolated by gas-chromatography from equilibrium mixtures ${ }^{25}$ obtained by acid catalysis or pyrolysis of the polymer or by hydrolysis of dichlorodimethylsilane. All members from the trimer to the hexadecamer have been isolated and characterized, and six further

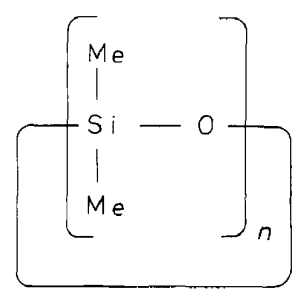

$n=3-16$

Figure 21 


\section{J. DALE}

members identified analytically. The questionable assumption ${ }^{1}$ that these ring skeletons would also try to follow the diamond lattice, in spite of the abnormally high valency angles at oxygen and presumably small rotational barriers in the SiO bonds, seemed to become justified by its ability to explain the alternation of the melting temperatures along the series (Figure 22). The argument is ${ }^{1}$ that only the oligomers having even $n$, particularly the octamer (16-membered ring), decamer, and so on, would have the same strain-free conformation in the solid as well as in the melt. These conformations should be of the general type shown in Figure 23 for the 16- and 20-membered ring.

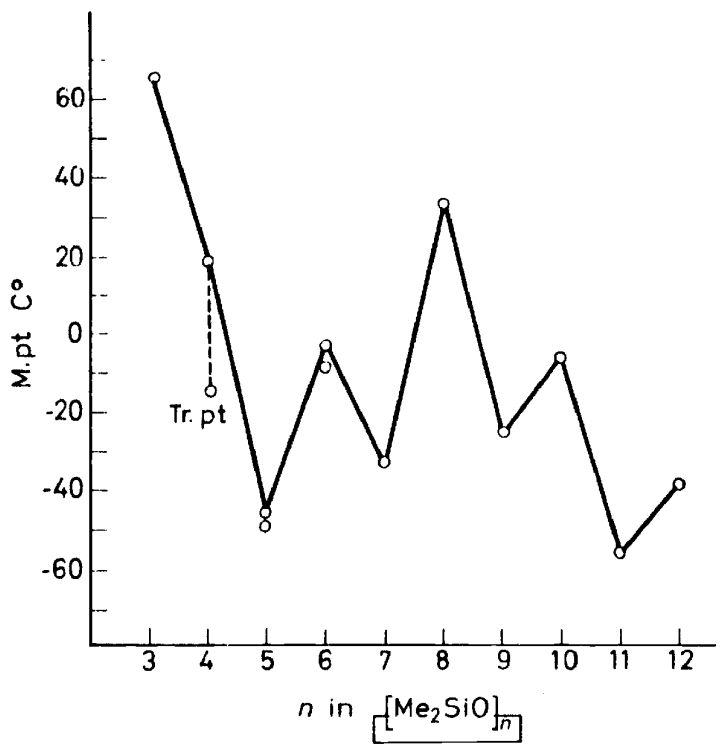

Figure 22

The former has a particularly high symmetry and has in fact been shown ${ }^{26}$ to be present in the crystal. However, the above argument is, strictly speaking, limited to a prediction of a low entropy of fusion with the underlying assumption that the enthalpy of fusion rises regularly with ring size. This is indeed true for many carbocyclic series ${ }^{1}$. However, when the heats of fusion were then determined calorimetrically, it turned out (Figure 24) that the values for these siloxanes alternated strongly and were particularly high for the octa- and decamer. Even more surprising, the entropies of fusion also alternated (Figure 25) and were highest, instead of lowest, for the same two oligomers. Since the maximum values occur for the octamer, which we know to possess a single well-defined and efficiently packed crystal conformation, it may perhaps be concluded that only this member, and possibly the decamer, undergoes the normal process of selecting for the crystal lattice one conformation out of several present in the liquid. The lower entropy of 
CONFORMATIONAL STUDIES OF SOME RING SYSTEMS<smiles>C[Si](C)(C)O[Si](C)(C)O[Si](C)(C)O[Si](C)(C)O[Si](C)(C)O[Si](C)(C)O[Si](C)(C)O[Si](C)(C)O[Si](C)(C)C</smiles>

(a)<smiles>C[Si](C)(C)O[Si](C)(C)O[Si](C)(C)O[Si](C)(C)O[Si](C)(C)O[Si](C)(C)O[Si](C)(C)O[Si](C)(C)O[Si](C)(C)O[Si](C)(C)O[As](C)(C)O[Si](C)(C)O[Si](C)(C)C</smiles>

(b)

Figure 23

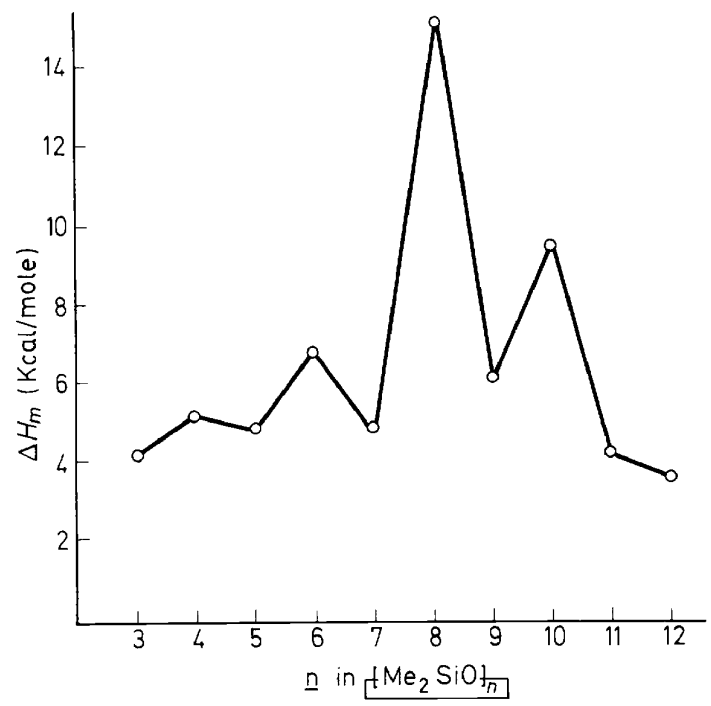

Figure 24

fusion for the other oligomers could then either mean that these are conformationally homogeneous, or, much more likely because of the accompanying low enthalpy of fusion, that the same mixture of conformers is present both in the crystal and in the liquid. No solid-phase transition point, at which conformational selection might occur, could be detected. For the tetra-, penta- and hexamer, transitions just below the melting point could be discerned, but the enthalpy and entropy changes are very small and have already been included with the values for the fusion process in Figures 24 and 25 . 


\section{J. DALE}

Mr Alvik then turned to the measurement of dipole moments hoping to learn more about the conformations in solution. A molecular dipole moment of zero can be predicted for the highly symmetric octamer crystal conformation (Figure 23), and relatively small moments for the other oligomers with even $n$. On the other hand, since no particularly favoured conformation can be envisaged for the oligomers with odd $n$, molecular dipole moments approaching a statistical (and higher) value are to be expected. The observed dipole moments in benzene solution (Figure 26) do show a weak alternation with ring size of the expected kind.

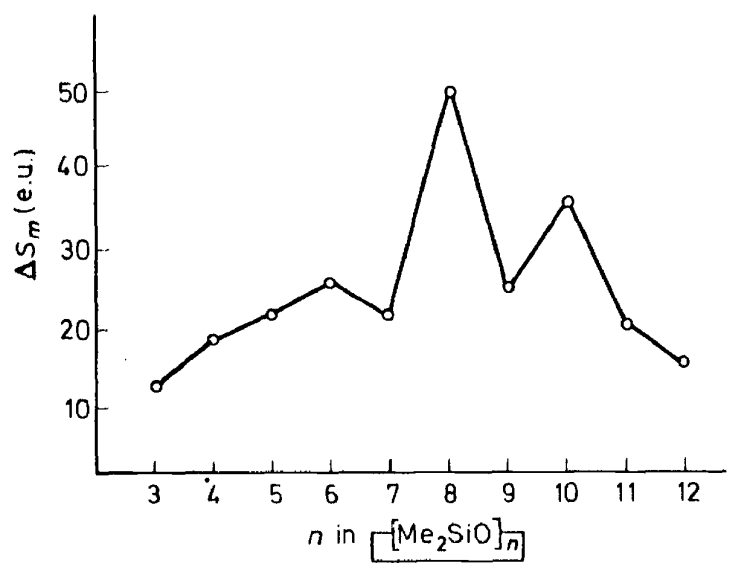

Figure 25

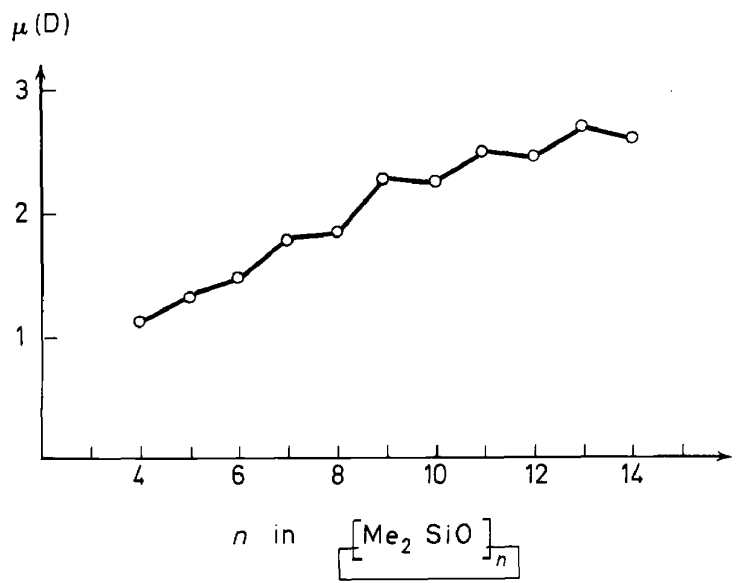

Figure 26 
But again, if the values, and the method used to calculate them from the experimental data, are critically scrutinized, the significance of this alternation becomes very doubtful. Using the Hedestrand procedure ${ }^{27}$, the total polarization was corrected only for the electronic polarization, obtained from the refractive index. We would expect the electronic polarization to increase regularly with ring size, and the alternation therefore to be already present in the total polarization. Instead we find that the total polarization shows no alternation, and that it is really introduced by an alternation present in the electronic polarization. What is much worse, since the electronic polarization for these compounds makes up as much as 75 per cent of the total polarization ${ }^{28}$, and we are not able to correct properly for the unknown atomic polarization, the error in the dipole moment may be enormous. A common procedure is to add 5-15 per cent to the electronic polarization, which is here more than doubtful, as the atomic polarization of the tetramer has been shown ${ }^{28}$ by microwave absorption to be particularly high, as much as 22 per cent of the total polarization, leaving only a few per cent, the difference between very large numbers, for the dipole orientation. This high contribution of the atomic polarization to the calculated apparent dipole moments in Figure 26 indicates an unusual flexibility, as do many other properties of siloxanes and the isoelectronic phosphonitrile halides ${ }^{1}$. Thus, the inversion of the $\mathrm{Si}-\mathrm{O}-\mathrm{Si}$ system is known to be very easy and can occur to produce the equivalent of an orientation of the net dipole moment. To cite Dasgupta. Garg and Smyth ${ }^{28}$, 'We enter a doubtful territory in which it may be difficult or arbitrary to distinguish between dipole orientation and atomic polarization'. The generally increasing moment along the series of cyclodimethylsiloxanes would suggest that in all these rings each monomer unit behaves as if it were independent and orients itself in the electric field. The resulting molecular conformation may then become very ill-defined, and a comparison with the static crystal-conformation, or the conformation resulting in solution from the influence of polar solvent molecules may be meaningless.

There are reasons to believe that the low basicity of the oxygen atom in siloxanes ${ }^{29}$ is connected with the large $\mathrm{Si}-\mathrm{O}-\mathrm{Si}$ angle, normally around $140^{\circ}$, and with the steric hindrance of the methyl groups. A variation of basicity along the oligomer series might then easily be produced by variations in molecular conformation. It was already known ${ }^{29}$ that the cyclic trimer, with $\mathrm{Si}-\mathrm{O}-\mathrm{Si}$ angle of only $136^{\circ}$, is more basic than higher oligomers. From n.m.r. studies of the hydrogen bonding between phenol and the siloxanes Mr Alvik could conclude that the tetramer and the hexamer, and possibly the octamer, are somewhat more basic than the penta-, hepta-, and nonamer. The oligomers of highest basicity are also those that gave the lowest electronic polarization. These properties may be connected, but it is impossible to draw any conclusion about molecular conformations.

Finally, it should be mentioned that the infra-red and Raman spectra show interesting progressions of low-frequency bands as ring size increases, strikingly resembling those observed for phosphonitrile halides ${ }^{30,31}$. This again stresses the similarity between these families of isoelectronic, highly flexible ring structures, but permits no conclusions about the conformations of the individual rings. 


\section{J. DALE}

\section{REFERENCES}

1 J. Dale. Angew. Chem. 78, 1070 (1966).

${ }^{2}$ G. J. Janz. Thermodynamic Properties of Organic Compounds, Academic Press, New York, 1967.

3 J. Dale, T. Ekeland and J. Schaug. Chem. Commun. 1477 (1968).

4 B. W. Roberts, J. J. Vollmer and K. L. Servis. J. Amer. Chem. Soc. 90, 5264 (1968).

${ }^{+4}$ A. Feigenbaum and J. M. Lehn. Bull. Soc. Chim. France. 3724 (1969).

5 J. Dale. Tetrahedron. 22, 3373 (1966).

${ }^{6}$ F. A. L. Anet and M. Z. Haq. J. Amer. Chem. Soc. 87, 3147 (1965).

${ }^{7}$ F. A. L. Anet and A. J. R. Bourn. J. Amer. Chem. Soc. 89, 760 (1967).

8 A. Bondi. Ann. N.Y. Acad. Sci. 53 II, 870 (1951).

9 J. B. Hendrickson. J. Amer. Chem. Soc. 89, 7047 (1967).

10 J. D. Dunitz and H. Eser. Helv. Chim. Acta. 50, 1565 (1967).

${ }^{11}$ H. Friebolin, W. Faisst, H. G. Schmid and S. Kabuss. Tetrahedron Letters, 1317 (1966).

12 N. J. Leonard and C. W. Schimelpfenig. J. Org. Chem. 23, 1708 (1958).

13 J. B. Hendrickson. J. Amer. Chem. Soc. 89, 7036 (1967).

14 J. D. Dunitz and H. M. M. Shearer. Helv. Chim. Acta. 43, 18 (1960).

15 A. T. Blomquist and R. D. Spencer. J. Amer. Chem. Soc. 70, 30 (1948).

${ }^{16}$ C. P. Smyth. Dielectric Behaviour and Structure, p. 371, McGraw-Hill. New York, (1955).

17 H. F. Kay and B. A. Newman. Acta Cryst. B24, 615 (1968).

18 G. Borgen and J. Dale. Chem. Commun. 447 (1969).

19 J. Dale and R. Coulon. J. Chem. Soc. 182 (1964).

${ }^{20}$ Huang-Minlon. J. Amer. Chem. Soc. 68, 2487 (1946).

21 J. Dale and K. Titlestad. Chem. Commun. 656 (1969).

22 M. Rothe, K. D. Steffen and I. Rothe. Angew. Chem. 77, 347 (1965).

${ }^{23}$ N. J. Leonard, J. A. Adamcik, S. Djerassi and O. Halpern. J. Amer. Chem. Soc. 80, 4858 (1958).

24 W. Fedeli and J. D. Dunitz. Helv. Chim. Acta, 51, 445(1968).

25 J. B. Carmichael, D. J. Gordon and F. J. Isackson. J. Phys. Chem. 71, 2011 (1967).

${ }^{26}$ L. K. Frevel and M. J. Hunter. J. Amer. Chem. Soc. 67, 2275 (1945).

27 G. Hedestrand. Z. Phys. Chem. B2, 428 (1929).

${ }^{28}$ S. Dasgupta, S. K. Garg and C. P. Smyth. J. Amer. Chem. Soc. 89, 2243 (1967).

${ }^{29}$ R. West, L. S. Whatley and K. J. Lake. J. Amer. Chem. Soc. 83, 761 (1961).

30 A. C. Chapman, N. L. Paddock, D. H. Paine, H. T. Searle and D. R. Smith. J. Chem. Soc. $3608(1960)$.

31 A. C. Chapman and N. L. Paddock. J. Chem. Soc. 635 (1962). 